\title{
Covalent CDK7 Inhibitor THZI Inhibits Myogenic Differentiation
}

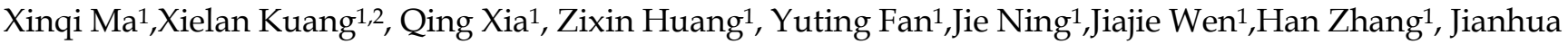 \\ Yan ${ }^{1}$, Qingjiong Zhang1, Huangxuan Shen ${ }^{1,2}{ }^{\bowtie}$, and Chongde Long ${ }^{1}$ \\ 1. State Key Laboratory of Ophthalmology, Zhongshan Ophthalmic Center, Sun Yat-sen University, 54 Xianlie Road, Guangzhou 510060, China. \\ 2. Biobank of Eye, State Key Laboratory of Ophthalmology, Zhongshan Ophthalmic Center, Sun Yat-sen University, 54 Xianlie Road, Guangzhou 510060, \\ China \\ $\triangle$ Corresponding authors: Huangxuan Shen, Ph.D., Zhongshan Ophthalmic Center, Sun Yat-sen University, 54 Xianlie Road, Guangzhou 510060, China. Phone: \\ (+8620)-8733-5261; FAX: (+8620)-8733-3271; Email: shenhx@mail.sysu.edu.cn. Chongde Long, Ph.D., M.D., Zhongshan Ophthalmic Center, Sun Yat-sen \\ University, 54 Xianlie Road, Guangzhou 510060, China. Phone: (+8620)-8733-1537; Email: longchd@mail.sysu.edu.cn. \\ (C) Ivyspring International Publisher. This is an open access article distributed under the terms of the Creative Commons Attribution (CC BY-NC) license \\ (https://creativecommons.org/licenses/by-nc/4.0/). See http://ivyspring.com/terms for full terms and conditions.
}

Received: 2018.02.06; Accepted: 2018.06.07; Published: 2018.08.06

\begin{abstract}
Covalent CDK7 inhibitor THZ1 is a newly discovered anti-tumor drug.THZ1 affects the function of transcription factor TFIIH by inhibiting CDK7, which in turn affects RNA polymerase II, and ultimately affects transcription initiation. Study found that THZI could inhibit proliferation and promote apoptosis of several tumor cell lines. However, there is no report of the potential side effect of THZ1 in normal tissues. In the course of cancer, the muscle consumption of cachexia needs to be supplemented by the differentiation of muscle cells. However, the effect of THZI on myogenic differentiation remains unclear. Our study in this article found that $\mathrm{THZ} 1$ could both inhibit the differentiation of $\mathrm{C} 2 \mathrm{Cl} 2$ cells and mouse primary myoblasts, also repressing the expression of differentiation-related transcription factors and muscle structural proteins, such as and myogenin, myh3 and MCK. Moreover, THZ1 could inhibit $\mathrm{C} 2 \mathrm{Cl} 2$ cell proliferation and migration, increase its oxidative stress and promote its apoptosis. Our data indicates that THZ1 inhibits myogenic differentiation, suggesting that therapies based on $\mathrm{THZ} 1$ might have potential side effects on muscle functions.
\end{abstract}

Key words: THZ1; Cachexia; C2C12 cells; myogenic differentiation

\section{Introduction}

Cachexia is a major complication of patients with various malignancies, and muscle depletion is critical to causing and exacerbating cachexia. Muscle consumption is the result of decreased synthesis and increased breakdown of muscle proteins, and nutrients supplementary could not improve this state of consumption [1, 2]. However, skeletal myogenesis is a complicated physiological process orchestrated by intracellular interactions and extracellular factors [3]. Satelliate cells lying beneath the basic lamina of muscle specifically express pax7, announcing its potential of myogenesis [4]. Pax7 then activates the expression of Myf5 and later MyoD, determining the cell as myogenic precusors in vivo and primary myoblasts in vitro [5-7]. After that, myoblasts and precusors who express myogenin and myosin heavy chain differentiate into myocytes [5]. Mononucleate myocytes fuse with each other, forming the multinucleate myotube, who has a contractile function [8].

Some transcription factors participate in this procedure, mainly myogenic regulatory actors (MRFs) and myocyte enhancer factors (MEFs) [9]. MRFs, including Myf5, MyoD, myogenin and MRF4, all belong to basic helix-loop-helix (bHLH) transcription family and targets E-box on DNA with its conserved sequence [10, 11]. Myf5 and MyoD express in proliferating period and gain their transient peak shortly after differentiation induction. When differentiation activity initiates, myogenin expression arises, which is modulated by MyoD [5].After several days of differentiation, myosin heavy chain expresses as a marker of muscle fibre [12].It's worth mentioning that expression of Myh and Mylpf is partly regulated by MyoD and myogenin. Finally, Myh and Mylpf act as heavy chain and light chain of fast skeletal muscle 
[4].Mef2 proteins, in which Mef2c is muscle-restricted, coordinate with MRFs in the binding of E-box [13]. Recently, more and more small-molecule compounds and medicines, even clinical drugs have been proved to be effective to influence myogenic differentiation, such as curcumin, ranolazine, dexamethasone, who provide new entry point towards the treatment of myopathies $[3,14,15]$.

THZ1 is a covalent inhibitor of cyclin-dependent kinase 7 (CDK7) [16].CDK7 has two roles, one is a part of transcriptional factor TFIIH, another is a member of CDK Activating Kinase (CAK) [17]. Correspondingly, CDK7 impacts on transcriptional initiation through phosphorylating serine-5 (S5) on the CTD of RNA Pol II as well as affecting cell cycle via activating other cyclin-dependent kinases such as CDK1 and CDK2 $[18,19]$. Resent researches put particular emphasis on the function of transcriptional inhibition of THZ1. Interestingly, THZ1 globally inhibits transcription but specifically inactivates CDK7 by covalently and irreversibly binding to a crsteine residue outside the CDK7 canonical kinase domain [20]. In the past few years, the anti-tumor role of THZ1 has gained more and more attention. THZ1 could induce apoptosis and impair the proliferation of Diffuse Intrinsic Pontine Glioma, small cell lung cancer and triple-negative breast cancer in vitro and in vivo [21-23]. It's well to be reminded that the suppression effect of THZ1 is likely to act towards genes that have a subtype of transcriptional enhancers called "super-enhancers" [24]. At present, it's indispensable to find a proper concentration between efficacy and toxicity so as to put THZ1 into clinical use [18]. Because of the wide-range inhibition of transcription, effect on other cell behaviors is waiting to be explored. Nevertheless, the impact of THZ1 on cell differentiation is almost unknown, especially myogenic differentiation. THZ1 is an anti-tumor drug with clinical application prospect, but its application should also consider the impact on cachexia process in tumor patients, especially on the biosynthesis of muscle.

Our study aims at exploring if THZ1 participate in myogenic differentiation. We found apparent inhibiting effect on myogenesis by non-toxic concentration of THZ1.

\section{Materials and methods}

\subsection{Reagents and plasmids}

THZ1 was purchased from Selleck Chemicals (USA, s7549).

For western blot, Myogenin antibody (1:1000, Absin), myosin heavy chain antibody (1:400, BOSTER Biological Technology Co.Ltd, BM4903) and $\beta$-tubulin antibody (1:1000, Affinity, USA), and the secondary rabbit antibody (1:6000, KangChen Bio-tech, KC-RB-035) were used.

The Myog-luc reporter gene [25] plasmid, MCK-luc reporter gene [26] plasmid and3 $\times$ MEF2-luc reporter gene plasmid [27] were used for luciferase assay.

\subsection{Cell culture and differentiation induction}

C2C12 cells (ATCC) was cultured in DMEM medium. The culture and differentiation conditions were following the before [11, 28].

\subsection{Scratch assay, Real-time RT-PCR and Luciferase assay}

All of four assays were performed as described previously [11, 28-30]. The C2C12 cells were cultured in a 6-well plate and used for the scratch assay when density reached $60 \%$.

We utilized Real-time RT-PCR to define the relative expression level of following genes: Myog (myogenin), Mylpf (myosin light chain), Myh3 (myosin heavy chain 3), MCK (muscle creatine kinase), Mef2c, and GAPDH acted as control. The primers were synthesized by SangonBiotech (Guangzhou). The sequence of forward and reverse chains was the same as described previously $[11,28$, 29].

\subsection{Apoptosis measurement, ROS assay and Cell Viability}

Using the methods described previously [11, 28, 29], the apoptosis of $\mathrm{C} 2 \mathrm{C} 12$ cells with or without THZ1 was measured by the Multicaspase Kit (Millipore). ROS was detected by the dihydroethidium (DHE; Millipore, EMD Millipore Corporation, Hayward,CA). We determined by MTT (3-(4,5-dimethylthiazol-2-yl)-2,5-diphenyl-tetrazolium bromide) method to measure cell viability.

\subsection{Mouse primary myoblasts}

The isolation and culture of primary myoblasts were performed as described previously [31]. The myoblasts were maintained in growth medium with $20 \%$ heat inactive fetal bovine serum.

\subsection{Statistical analysis}

Each experiment was repeated at least three times, using IBM SPSS Statistics 20 software for statistical analysis. The results are displayed as mean \pm standard deviation, the significance of the differences among groups were determined by multiple LSD's multiple-comparison test(one-way ANOVA).Significant $P$ values are shown as $P<0.05$ $(*), P<0.01(* *), P<0.001(* * *)$ and $P>0.05(\#)$. 


\section{Results}

\section{THZ1 induced $C 2 C 12$ cell apoptosis and inhibited $\mathrm{C} 2 \mathrm{C} 12$ cell proliferation.}

We could determine cell growth by apoptosis and proliferation. At first, we tried to figure out how THZ1 affected apoptosis. Through staining cell nucleus with Multicaspase Reagent and 7-AAD after treating cells with gradient concentration differing from 0 to $800 \mathrm{nM}$ for $24 \mathrm{~h}$, we calculated cell apoptosis with flow cytometry assay (Fig. 1A,1B). Apoptosis started becoming obvious from concentration 400 in a dose-dependent manner. The induction effect below concentration 200 had no significance compared to control, that's to say, only relatively high concentration could induce cell apoptosis of C2C12 cells. Then we tried to explore if intracellular reactive oxygen species (ROS) contributes to the apoptosis of cells in vitro (Fig. 1C,1D). We found that the count of ROS+ cell grew significantly more from concentration 200nM. We carried out MTT assay to test the impact of THZ1 on proliferation of $\mathrm{C} 2 \mathrm{C} 12$ cell in vitro. We treated $\mathrm{C} 2 \mathrm{C} 12$ cells with gradient concentration of THZ1 from 0 to $800 \mathrm{nM}$ and calculated OD value in 0 h, $24 \mathrm{~h}, 48 \mathrm{~h}$ and $72 \mathrm{~h}$ (Fig. 1E). At each time nodes, OD values of concentration 25,50, 100 treated groups are almost similar to those control groups, reflecting the unaffected cell viability under the concentration above. From concentration $200 \mathrm{nM}$ to $600 \mathrm{nM}$, cell viability was gradually descending at three time points respectively. On account of the MTT assay, apoptosis assay and ROS assay above, we decided to choose concentration $0 \mathrm{nM}, 50 \mathrm{nM}$ and $100 \mathrm{nM}$ to perform scratch assay and differentiation assay following, because of their relative no-damage effect towards $\mathrm{C} 2 \mathrm{C} 12$ cells. In addition to $\mathrm{C} 2 \mathrm{C} 12$, in order to observe the effect of THZ1 on tumor cells and normal cells apoptosis, we selected MC38 cancer cells as well as mouse E14 cells and 661w cells as control. The level of apoptosis was detected after the cells were treated with different concentration of THZ1. Apoptosis of MC38 cancer cells significantly increased and cell vitality significantly reduced from the concentration of $400 \mathrm{nM}$ (Figure S1A,1B,1G). Apoptosis of E14 cells wasn't increased until the concentration of THZ1 reached $800 \mathrm{nM}$ (Figure S1C,1D), while in $661 \mathrm{~W}$ cells, the apoptosis concentration was still $800 \mathrm{nM}$ (Figure $\mathrm{S} 1 \mathrm{E}, 1 \mathrm{~F})$.However, the apoptosis of $\mathrm{C} 2 \mathrm{C} 12$ cells began from $400 \mathrm{nM}$, indicating that treatment concentration of THZ1 affected proliferation of $\mathrm{C} 2 \mathrm{C} 12$ cells , which also belongs to side effects of THZ1.

\section{A}
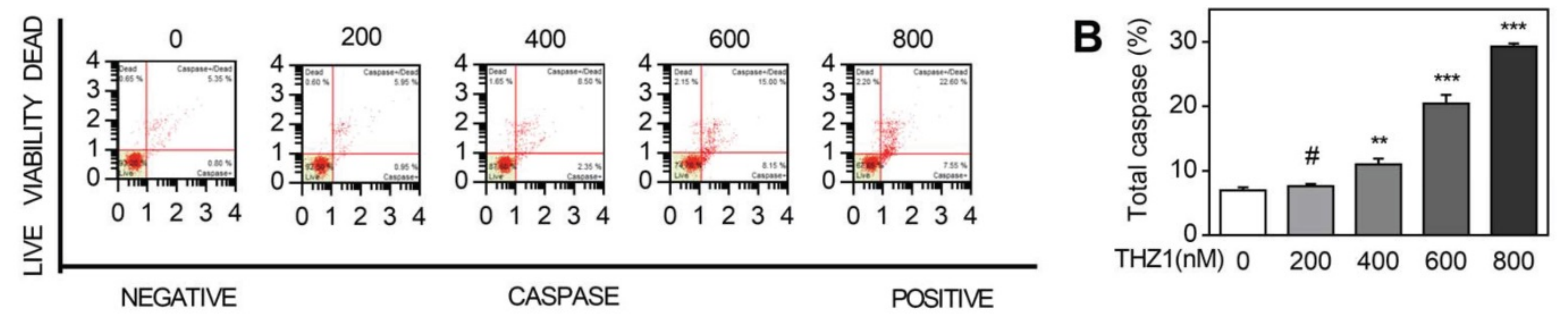

C
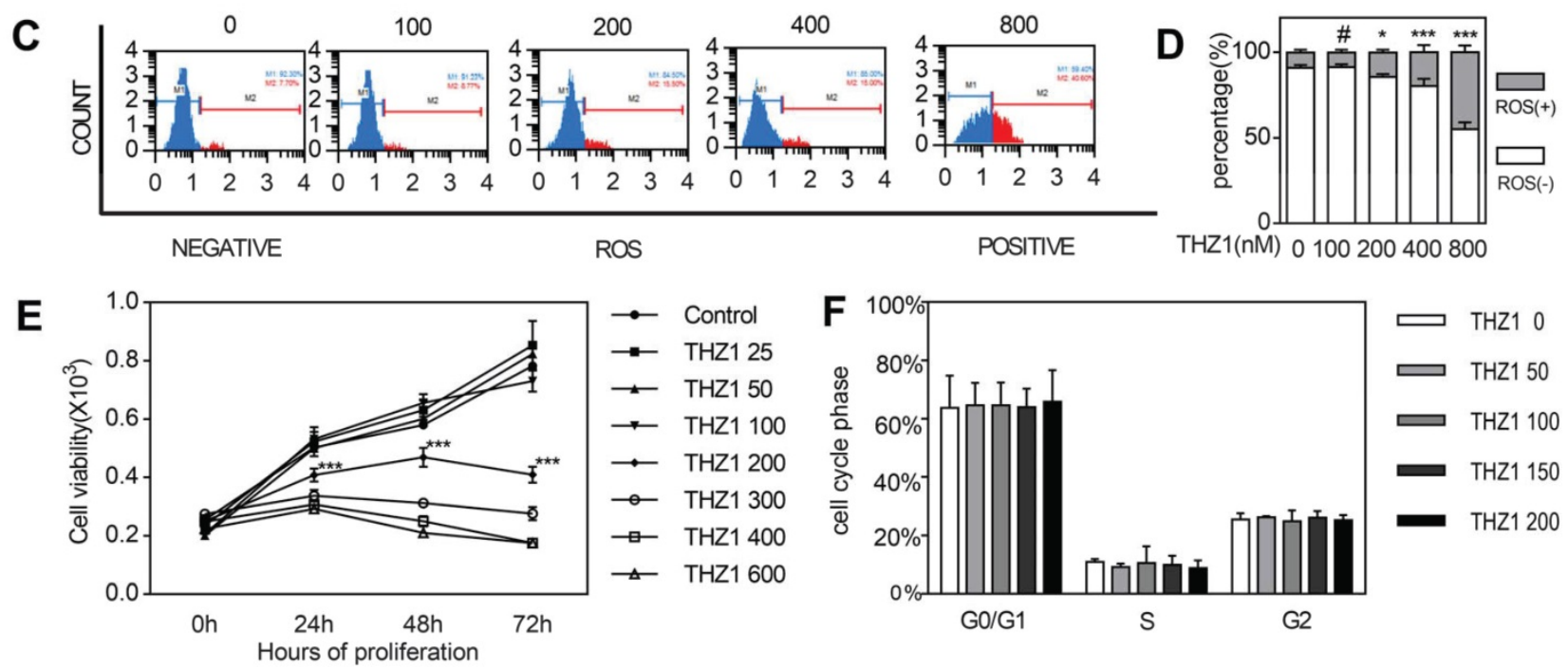

Figure 1. THZ1 induced $\mathrm{C} 2 \mathrm{C} 12$ cell apoptosis and inhibits $\mathrm{C} 2 \mathrm{C} 12$ cell proliferation. (A)C2C12 cells were treated with gradient concentration of THZ1 $(0,200,400,600,800 \mathrm{nM})$ for $24 \mathrm{~h}$. The percentage of live and apoptosis cells were measured on flow cytometry.(B)Independent experiment was repeated for three times. Bar graph is for quantification of figure 1A.(C) C2C12 cells were treated with gradient concentration of THZ1 $(0,100,200,400,800 \mathrm{nM})$ for $24 \mathrm{~h}$.Intracellular ROS were measured on flow cytometry.(D) Independent experiment was repeated for three times. Bar graph is for quantification of figure $1 \mathrm{C}$.(E)OD values in MTT assay were measured after C2C12 cells were treated with gradient concentration of THZ1 $(0,25,50,100,200,300,400,600 \mathrm{nM})$ for $24 \mathrm{~h}, 48 \mathrm{~h}, 72 \mathrm{~h}$ respectively. $(\mathrm{F}) \mathrm{C} 2 \mathrm{Cl} 2 \mathrm{cells}$ were incubated for $24 \mathrm{~h}$ in different concentration $(0,50,100,150,200 \mathrm{nM})$ and cell cycle was determined on flow cytometry. $(\# P>0.05, * P<0.05, * * P<0.01, * * * P<0.001, n=3$, bars represent $S D)$. 


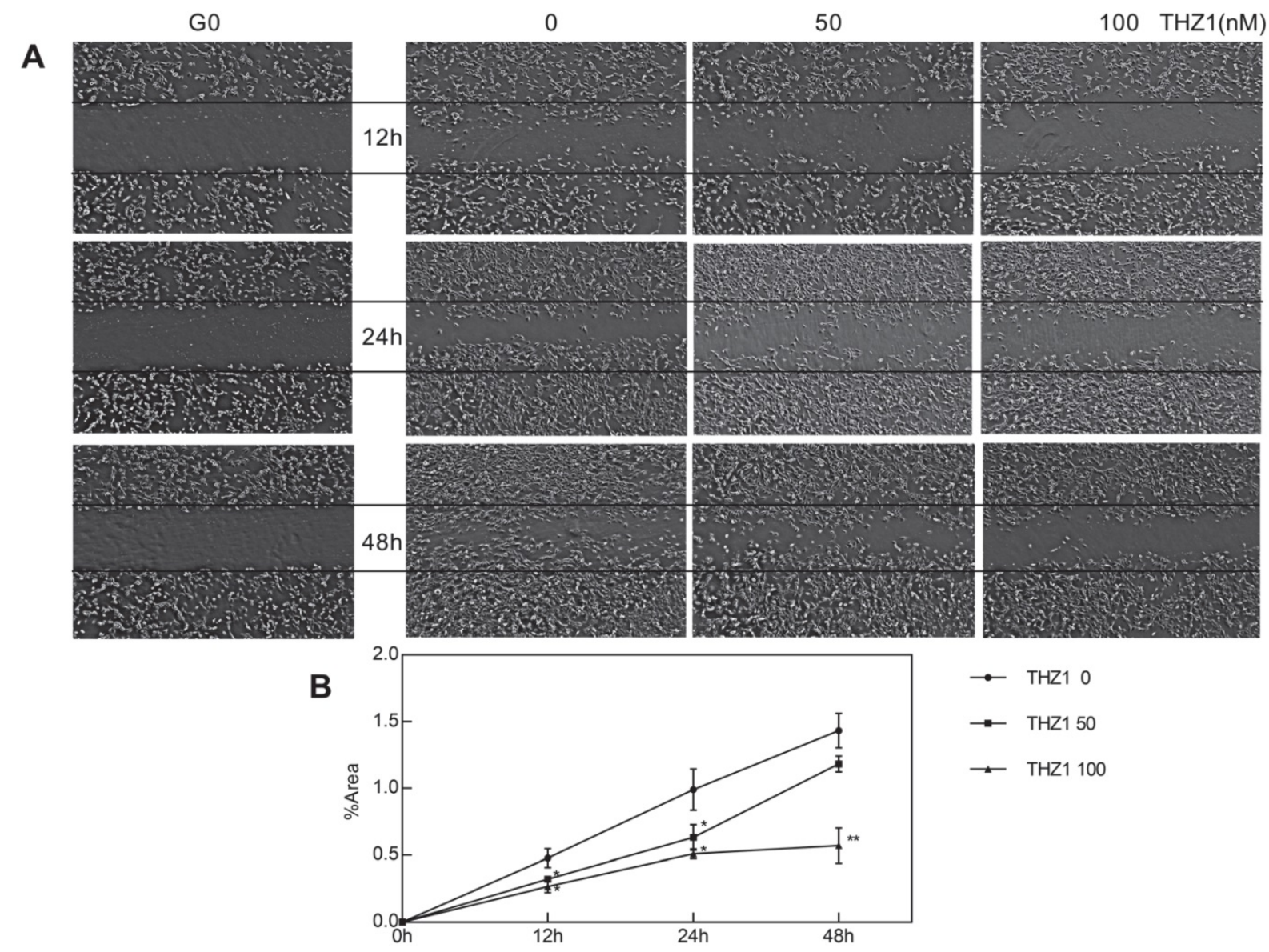

Figure 2. THZ1 inhibited migration of $\mathrm{C2C12}$ cells. (A)Six scratch wounds were made per disk when cell density reached $60 \%$. Growing medium was changed with medium of $3 \% \mathrm{FBS}$ and $1 \%$ penicillin and $\mathrm{C} 2 \mathrm{Cl} 2$ cells were treated with gradient concentration $(0,50,100 \mathrm{nM})$. Pictures were taken in $12 \mathrm{~h}, 24 \mathrm{~h}, 48 \mathrm{~h}$ respectively, among which pictures of $\mathrm{C} 0$ were taken immediately after the scratch was created.(B)(C)(D) Area taken by cells inside the two black lines was calculated by image. Nine cross points each picture was put into calculation and independent experiment was repeated for three times. $(* P<0.05, * * P<0.01, n=3$, bars represent $S D)$.

\section{THZ1 inhibited migration of $\mathrm{C} 2 \mathrm{C} 12$ cells}

We wondered whether other biological behaviors of $\mathrm{C} 2 \mathrm{C} 12$ cells would be affected, particularly migration and differentiation, at a relatively low concentration that did not affect $\mathrm{C} 2 \mathrm{C} 12$ cell apoptosis and proliferation. After planting $\mathrm{C} 2 \mathrm{C} 12$ cell at the density of $60 \%$ and creating scratch, we took photos at the time point $0 \mathrm{~h}, 12 \mathrm{~h}, 24 \mathrm{~h}, 48 \mathrm{~h}$ respectively (Fig. 2A). At first, we calculated cell cover area of each picture and found no significant difference, verifying again that cell proliferation wasn't influenced. At each time point, the scratch of THZ1 treated group owned larger area, showing poorer healing ability compared to control group in a dose-dependent manner (Fig. 2B). However, the wound-healing ability of THZ1 $100 \mathrm{nM}$ treated group didn't lose totally and until $48 \mathrm{~h}$, the migrating activity was proceeding continuously as time went.

\section{Low concentration of $\mathrm{THZ} 1$ inhibited $\mathrm{C} 2 \mathrm{C} 12$ cell differentiation}

To prove that THZ1 has the ability to inhibit $\mathrm{C} 2 \mathrm{C} 12$ cells differentiation, we verified from different aspects. The first was morphology. As the most intuitive method, we took pictures in the third day of differentiation (Fig. 3A). For the control group, the cells were in the middle and late stages of differentiation, and the mononuclear cells gradually merged into multi-nuclear myotubes. The morphology of the cells switched from spindle to a long bar. Our results showed that compared with the control group, the number of differentiated cells in the same area of vision decreased in the treatment group, and the cells did not undergo fusion to the direction of differentiation and differentiation ability decreased with increasing concentration. Realtime RT-PCR was then verified from mRNA levels transcribed from the genes (Fig. 3B). Whether in the first day or the third day of differentiation, the structural genes Myh3 and Mylpf both decreased with the increase of the drug concentration, meanwhile the drug also inhibited the expression of transcription factors Myogenin and MCK and at a drug concentration of 50 , that is, at a relatively low concentration, inhibition of transcription had emerged. What's deserved to be mentioned is that, compared with the RNA expression of myogenin, MCK, Mylpf and Myh3in the 
first day, in the third day. The ratio of relative mRNA expression of concentration 50 and concentration 0 descended, indicating the inhibiting effect started becoming more obvious in late differentiation stage. Furthermore, to explore the promoter activity of differentiation marker genes including MCK, Mef2c and Myogenin, we performed a luciferase assay (Fig. 3C). When compared with the expression of Renilla fluorescein, we concluded that the drug disrupted the normal regulatory effect of the transcription factor on the promoter and decreased the priming of the gene of interest. And at a drug concentration of 50, that is, at a relatively low concentration, inhibition of transcription had emerged. At the protein level, we performed western blot of differentiation marker Myogenin and Myh3 and the result verified the former consequences (Fig. 3D, 3E, 3F, 3G). However, in order to verify whether such inhibition of differentiation occurs before it plays an anti-tumor role, we selected mouse colon cancer cells from the same species of $\mathrm{C} 2 \mathrm{C} 12$ cells as a control. After adding gradient concentration of THZ1, through detection of cell apoptosis and proliferation, we found that apoptosis of cancer cells significantly increased and cell vitality significantly reduced from the concentration of $400 \mathrm{nM}$ (Figure S1A, 1B, 1G).

\section{THZ1 inhibits differentiation of mouse primary myoblasts}

Primary cultured myoblasts were harvested from gastrocnemius and quadriceps muscle of 3-week-old mice and induced to differentiate. One day later, the expression of Myogenin, Mef2c and Myh3 and Mylpf were detected by qRT-PCR (Fig. 4A). The experimental group used THZ1 concentrations of 50 and 100. The results showed that with the drug concentration increased, the expression of the above four genes decreased. At gene start level, we
A
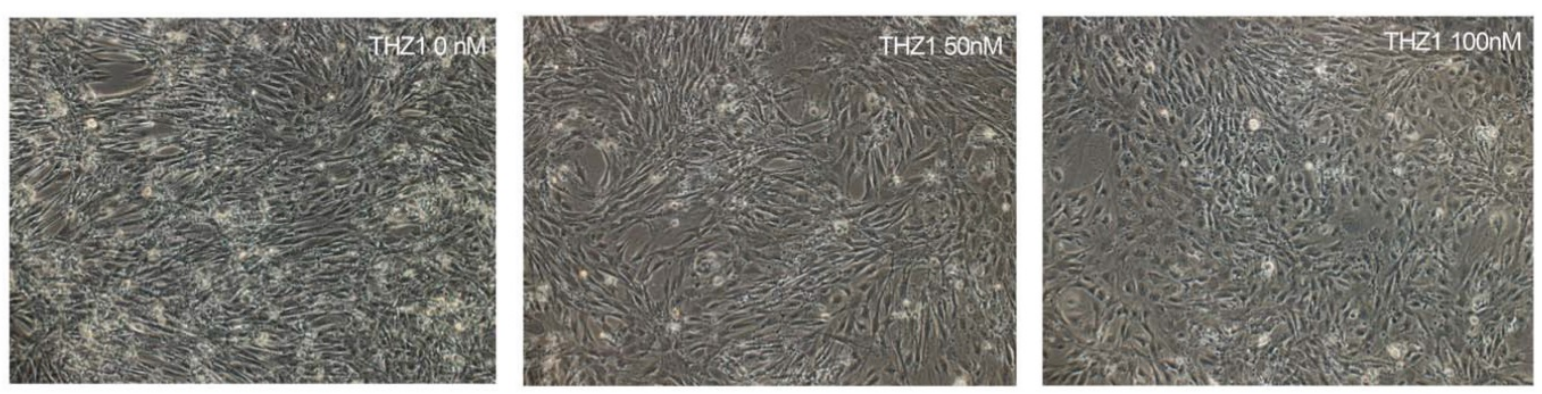

B
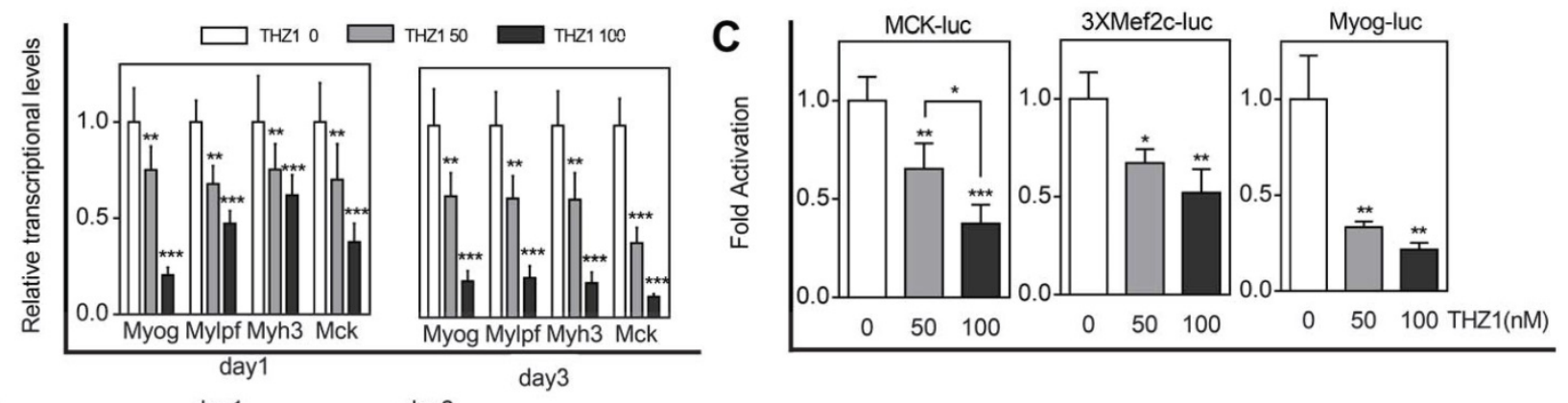

D

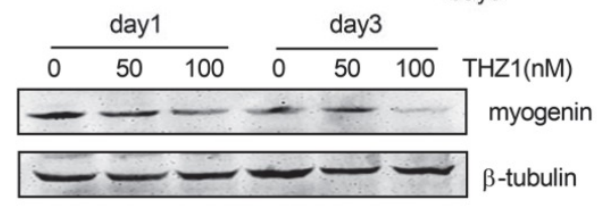

E

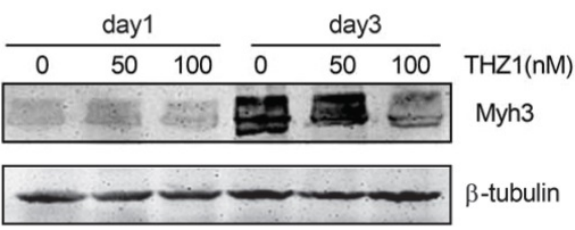

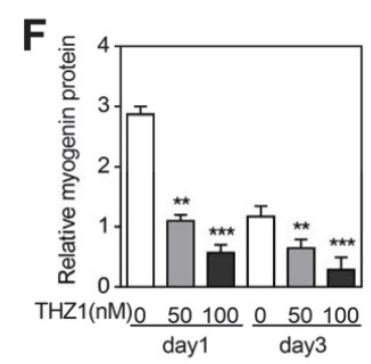

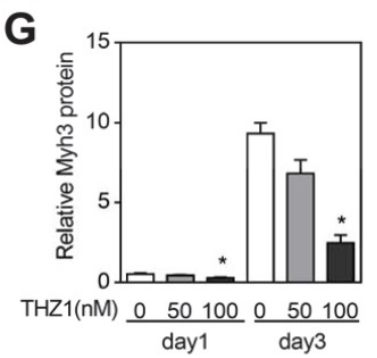

Figure 3. Low concentration of THZ1 inhibited $\mathrm{C} 2 \mathrm{Cl} 2$ cell differentiation. (A) $C 2 \mathrm{C} 12$ myoblasts were induced to differentiate and meanwhile treated by gradient concentrations of THZ1 (0,50, $100 \mathrm{nM})$. Pictures were taken three days later. (B) qRT-PCR was performed to analyze the transcript levels of muscle-specific genes including Myog, Myh3, Mylpf and MCK. RNA samples were collected one and three days after differentiation respectively. (C)MCK-luc, 3xMef-luc and Myog-luc were co-transfected with pEF-RL respectively. 24h after transfection, gradient concentration of THZ1 (0, 50, $100 \mathrm{nM})$ was added. $48 \mathrm{~h}$ after differentiation induction, luciferase activity was measured.(D)(F) One and three days after differentiation induction, protein level of Myh3 and Myogenin were analyzed by western blot.(E) Bar graph was gray intensity analysis of figure 3D. Gray values were normalized to $\beta$-tubulin.(G) Bar graph was gray intensity analysis of figure $3 \mathrm{~F}$. Gray values were normalized to $\beta$-tubulin. $(* P<0.05$, $* * P<0.01$, $* * * P<0.001, n=3$, bars represent SD). 

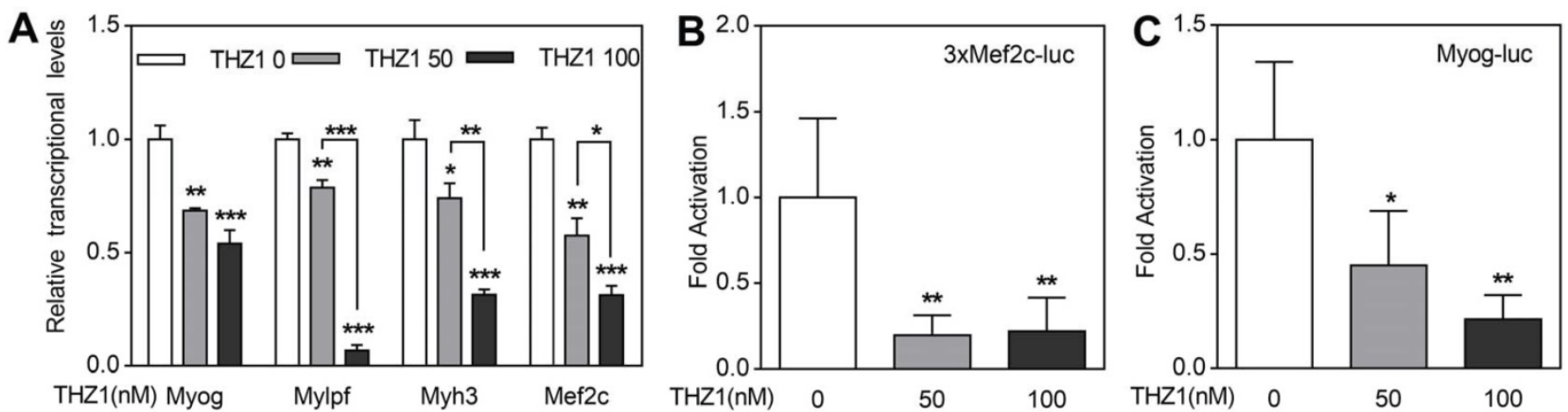

D
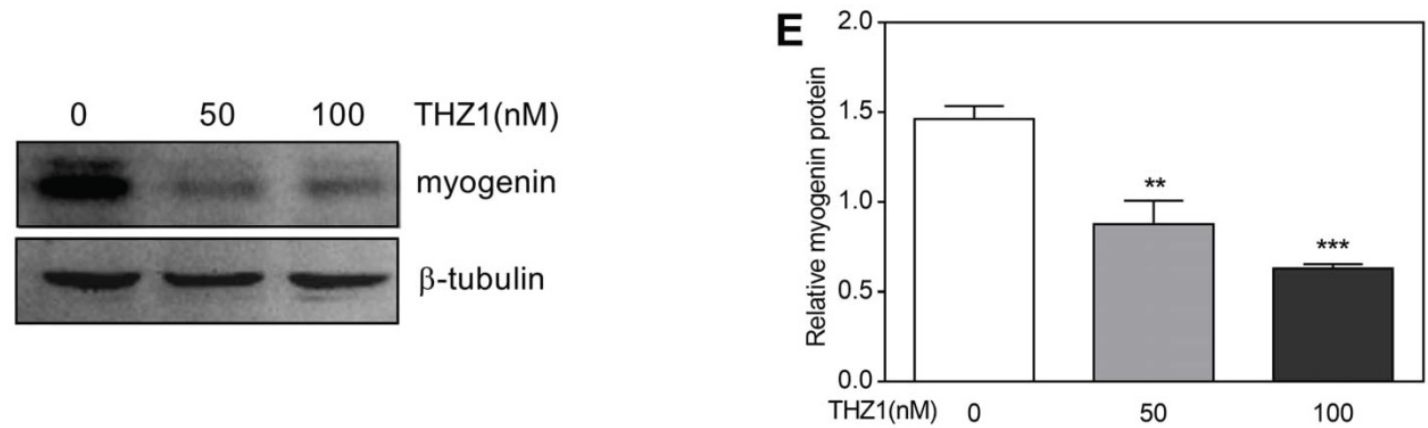

Figure 4. THZ1 inhibited differentiation of mouse primary myoblasts. (A) In mouse primary myoblasts, qRT-PCR was performed to analyze the transcription levels of muscle-specific genes including Myog, Myh3, Mylpf and Mef2c.(B) 3xMef-luc and Myog-luc were co-transfected with pEF-RL respectively. $24 \mathrm{~h}$ after transfection, gradient concentration of THZ1 $(0,50,100 \mathrm{nM})$ was added. $48 \mathrm{~h}$ after differentiation induction, luciferase activity was measured. (C) Protein level of myogenin in mouse primary myoblast was analyzed by western blot. (D) Bar graph was gray intensity analysis of figure $4 C$. Gray values were normalized to $\beta$-tubulin. $(* P<0.05$, $* * P<0.01, * * * P<0.001, n=3$, bars represent SD).

performed luciferase on Mef2c and Myogenin, which are early markers of differentiation (Fig. 4B, 4C, 4D). Compared with Renilla fluorescein, we found that the activation of Mef2c and Myogenin in the drug-treated group decreased. At protein level, we performed western blot of early differentiation marker Myogenin and the result verified the former consequences (Fig. $4 \mathrm{E}, 4 \mathrm{~F})$.

\section{Discussion}

Our study, for the first time, found that THZ1 inhibits the differentiation of $\mathrm{C} 2 \mathrm{C} 12$ cells and primary myoblasts at relatively low concentrations, and at this concentration, the cell cycle isn't affected. Higher than the concentration of $400 \mathrm{nM}$, there's a significant increase in cell apoptosis. Higher than the concentration of $200 \mathrm{nM}$, the proliferation of cells was inhibited and intracellular oxidative stress increased. At concentrations of 50 and 100, the differentiation of C2C12 cells was inhibited. However, THZ1 is an anti-tumor drug, so we start to think about the most common complication in the course of the tumorcachexia. Cachexia often reduces the patient's ability to bear radiotherapy and chemotherapy [1]. It can be enlightened from our experimental results that the concentration choice of THZ1 has to take the side effects on the muscles into consideration and slow down the cachexia process. However, it's a long way for THZ1 to go into clinical application and animal experiments and clinical trials support are also in need.

In the scratch test, we observed that THZ1 at a concentration of 50 and at a concentration of 100 resulted in a decrease in cell migration ability. A decrease in the ability to migrate makes it difficult for cells to contact with each other, reducing the signal transduction between cells and thereby suppressing the progress and the outcome of differentiation. That THZ1 reduces cell-cell contact may be one of the mechanisms of differentiation inhibition.

THZ1 has a suppressive effect on proliferation in many cell lines [21-23]. We found that THZ1 can also inhibit $\mathrm{C} 2 \mathrm{C} 12$ cell proliferation and promote apoptosis. THZ1 is a covalent inhibitor of CDK7, which is a cell cycle related protein [20], however, we found that THZ1 has little effect on cell cycle; Secondly, as part of the transcription factor TFIIH, THZ1 inhibits RNA polymerase II by inhibiting TFIIH function, thus inhibiting the formation of transcription initiation complexes [32]. However, our experiments showed that THZ1 can not only inhibit the transcription of differentiation-related genes, but also inhibit the expression of differentiation-related proteins and change the outcome of differentiation events. We speculate that THZ1 affects the function of RNA polymerase II to inhibit differentiation.

Of course, the exact mechanism of THZ1 needs further experimental study. 


\section{Supplementary Material}

Supplementary figure.

http://www.jcancer.org/v09p3149s1.pdf

\section{Acknowledgements}

This work was sponsored by the National Natural Science Foundation of China (81670874) and the Fundamental Research Funds of the State Key Laboratory of Ophthalmology. MC38 cells were from Dr. Dan Xie (Cancer Center, Sun Yat-sen University). $661 w$ cells were kindly provided by Dr. Xiaobo Zhu (State Key Laboratory of Ophthalmology, Sun Yat-sen University). E14 cells were attained from Dr. Junjiu Huang (School of Life Sciences, Sun Yat-sen University). Thanks Dr. Shuibin Lin (The First Affiliated Hospital, Sun Yat-sen University) and Dr. Lizi Wu (Department of Molecular Genetics and Microbiology, University of Florida) for their guidance on our article.

\section{Competing Interests}

The authors have declared that no competing interest exists.

\section{References}

1. Daly LE, Ni Bhuachalla EB, Power DG, Cushen SJ, James K, Ryan AM. Loss of skeletal muscle during systemic chemotherapy is prognostic of poor survival in patients with foregut cancer. Journal of cachexia, sarcopenia and muscle. 2018; 9(2): 315-325.

2. VanderVeen BN, Fix DK, Carson JA. Disrupted Skeletal Muscle Mitochondrial Dynamics, Mitophagy, and Biogenesis during Cancer Cachexia: A Role for Inflammation. Oxidative medicine and cellular longevity. 2017; 2017: 3292087.

3. Ileana T, Anna M, Pamela S, Fernanda V, Stefano B, Livio L. Ranolazine promotes muscle differentiation and reduces oxidative stress in $\mathrm{C} 2 \mathrm{C} 12$ skeletal muscle cells. Endocrine. $2017 ;$ 58(1): 33-45.

4. Amann R, Wyder S, Slavotinek AM, Trueb B. The FgfrL1 receptor is required for development of slow muscle fibers. Developmental biology. 2014; 394: $228-41$.

5. Rudnicki MA, Le Grand F, McKinnell I, Kuang S. The molecular regulation of muscle stem cell function. Cold Spring Harbor symposia on quantitative biology. 2008; 73: 323-31

6. Buckingham M. Skeletal muscle progenitor cells and the role of Pax genes. Comptes rendus biologies. 2007; 330: 530-3.

7. Buckingham M. Myogenic progenitor cells and skeletal myogenesis in vertebrates. Current opinion in genetics \& development. 2006; 16: 525-32.

8. Montarras D, L'Honore A, Buckingham M. Lying low but ready for action: the quiescent muscle satellite cell. The FEBS journal. 2013; 280: 4036-50.

9. Perry RL, Rudnick MA. Molecular mechanisms regulating myogenic determination and differentiation. Frontiers in bioscience : a journal and virtual library. 2000; 5: D750-67

10. Montarras D, Chelly J, Bober E, Arnold H, Ott MO, Gros F, et al. Developmental patterns in the expression of Myf5, MyoD, myogenin, and MRF4 during myogenesis. The New biologist. 1991; 3: 592-600.

11. Chen Z, Jin G, Lin S, Lin X, Gu Y, Zhu Y, et al. DNA methyltransferase inhibitor CDA-II inhibits myogenic differentiation. Biochemical and biophysical research communications. 2012; 422: 522-6.

12. Tajsharghi H, Oldfors A. Myosinopathies: pathology and mechanisms. Acta neuropathologica. 2013; 125: 3-18.

13. Dong C, Yang X, Zhang C, Liu Y, Zhou R, Cheng Q, et al. Myocyte enhancer factor $2 \mathrm{C}$ and its directly-interacting proteins: A review. Progress in biophysics and molecular biology. 2017; 126: 22-30.

14. Mirza KA, Luo M, Pereira S, Voss A, Das T, Tisdale MJ. In vitro assessment of the combined effect of eicosapentaenoic acid, green tea extract and curcumin C3 on protein loss in C2C12 myotubes. In vitro cellular \& developmental biology Animal. 2016; 52: 838-45.

15. Han DS, Yang WS, Kao TW. Dexamethasone Treatment at the Myoblast Stage Enhanced C2C12 Myocyte Differentiation. International journal of medical sciences. 2017; 14: 434-43.

16. Posternak V, Cole MD. Strategically targeting MYC in cancer. F1000Research. 2016; 5 .
17. Coin F, Egly JM. Revisiting the Function of CDK7 in Transcription by Virtue of a Recently Described TFIIH Kinase Inhibitor. Molecular cell. 2015; 59: 513-4.

18. Franco HL, Kraus WL. No driver behind the wheel? Targeting transcription in cancer. Cell. 2015; 163: 28-30.

19. Compe E, Egly JM. Nucleotide Excision Repair and Transcriptional Regulation: TFIIH and Beyond. Annual review of biochemistry. 2016; 85: 265-90.

20. Cao K, Shilatifard A. Inhibit globally, act locally: CDK7 inhibitors in cancer therapy. Cancer cell. 2014; 26: 158-9.

21. Nagaraja S, Vitanza NA, Woo PJ, Taylor KR, Liu F, Zhang L, et al. Transcriptional Dependencies in Diffuse Intrinsic Pontine Glioma. Cancer cell. 2017; 31: 635-52 e6.

22. Augert A, MacPherson D. Treating transcriptional addiction in small cell lung cancer. Cancer cell. 2014; 26: 783-4.

23. Li B, Ni Chonghaile T, Fan Y, Madden SF, Klinger R, O'Connor AE, et al. Therapeutic Rationale to Target Highly Expressed CDK7 Conferring Poor Outcomes in Triple-Negative Breast Cancer. Cancer research. 2017; 77: 3834-45.

24. Chipumuro E, Marco E, Christensen CL, Kwiatkowski N, Zhang T, Hatheway $\mathrm{CM}$, et al. CDK7 inhibition suppresses super-enhancer-linked oncogenic transcription in MYCN-driven cancer. Cell. 2014; 159: 1126-39.

25. Ohto H, Kamada S, Tago K, Tominaga S, Ozaki H, Sato S, et al. Cooperation of Six and Eya in activation of their target genes through nuclear translocation of Eya. Molecular and Cellular Biology. 1999; 19: 6815-24.

26. Lazaro JB, Bailey PJ, Lassar AB. Cyclin D-cdk4 activity modulates the subnuclear localization and interaction of MEF2 with SRC-family coactivators during skeletal muscle differentiation. Genes \& Development. 2002; 16: 1792-805.

27. Lu JR, McKinsey TA, Zhang CL, Olson EN. Regulation of skeletal myogenesis by association of the MEF2 transcription factor with class II histone deacetylases. Molecular cell. 2000; 6: 233-44.

28. Shen H, McElhinny AS, Cao Y, Gao P, Liu J, Bronson R, et al. The Notch coactivator, MAML1, functions as a novel coactivator for MEF2C-mediated transcription and is required for normal myogenesis. Genes \& Development. 2006; 20: 675-88

29. Cole CB, Russler-Germain DA, Ketkar S, Verdoni AM, Smith AM, Bangert CV, et al. Haploinsufficiency for DNA methyltransferase 3A predisposes hematopoietic cells to myeloid malignancies. The Journal of clinical investigation. 2017; 127: 3657-74.

30. Fan Y, Huang Z, Long C, Ning J, Zhang H, Kuang X, et al. ID2 protects retinal pigment epithelium cells from oxidative damage through p-ERK1/2/ID2/NRF2. Archives of biochemistry and biophysics. 2018; 650: $1-13$.

31. Lin S, Shen H, Jin B, Gu Y, Chen Z, Cao C, et al. Brief Report: Blockade of Notch Signaling in Muscle Stem Cells Causes Muscular Dystrophic Phenotype and Impaired Muscle Regeneration. Stem Cells. 2013; 31: 823-8.

32. Ebmeier CC, Erickson B, Allen BL, Allen MA, Kim H, Fong N, et al. Human TFIIH Kinase CDK7 Regulates Transcription-Associated Chromatin Modifications. Cell reports. 2017; 20: 1173-86. 